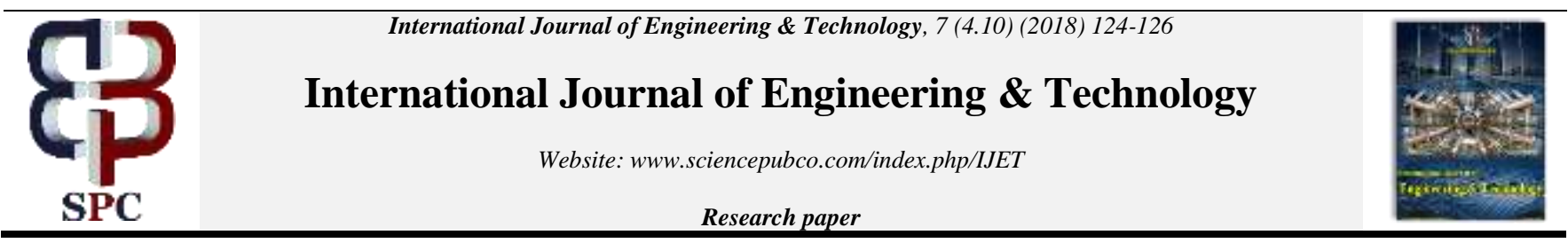

\title{
Domination Number of Rough Ideal based Rough Edge Cayley Graph
}

\author{
B. Praba ${ }^{1}$, X. A. B. Obilia ${ }^{2}$ \\ ${ }^{1}$ Department of Mathematics, SSN College of Engineering, Chennai-603110, India \\ ${ }^{2}$ Department of Mathematics, SSN College of Engineering, Chennai-603110, India \\ *Corresponding author E-mail: prabab@ssn.edu.in
}

\begin{abstract}
In this paper, we define a Cayley graph corresponding to the Rough ideal $J$ of the Rough semiring $(T, \Delta, \nabla)$. The Domination number of the Rough Ideal based Rough Edge Cayley graphs $G(T(J))$ and $G(T(\widehat{J}))$ where $\widehat{J}$ contains the non-trivial elements of J are derived and illustrated through examples. Interpretation of a communication network is done in the form of Rough Ideal based Rough Edge Cayley Graph.
\end{abstract}

Keywords: Rough set, Cayley graph, dominating set, Rough graph, Adjacency.

\section{Introduction}

The concept of Rough set theory was proposed by Pawlak, [10] to deal with the incomplete information system. Jinkun Chen and Jin Jin Li [3] have discussed on the applications of rough sets to graph theory. M.H. Shahzamanian, et.al [9] have discussed the roughness in Cayley graphs. Roughness based on fuzzy ideals was discussed by Davvaz [2]. The concept of Rough lattice was described by B.Praba and Mohan [6]. The concept of semiring on rough sets were discussed by B.Praba, et.al [7]. The concept of ideals on Rough sets have also been discussed by A.Manimaran, et.al. The characterization of Rough semiring was discussed by A.Manimaran, et.al [4]. V.Selvan and G. Senthil Kumar [8] have given a notion on the rough ideals of semiring.

The concept of Edge Rough graph and its applications were discussed by Meilian Liang, et.al [5].There are been many research works done on Algebraic graph theory. In our paper we give an algebraic graph theoretical approach to rough sets and discuss on the Rough Ideal based Rough Edge Cayley graphs [8]. This paper is organised as follows: In section 2, we give some preliminary definitions on Rough set theory, Graph Theory and Rough Ideal based Rough Edge Cayley graph. In section 3, we obtain the Domination number of any Rough Ideal based Rough Edge Cayley graph and illustrate it with examples. In section 4, we have interpreted a communication network in the form of a Rough Ideal based Rough Edge Cayley graph. In section 5, we give the conclusion.

\section{Preliminaries}

\subsection{Rough Set Theory}

In this section we consider an approximation space $\mathrm{I}=(\mathrm{U}, \mathrm{R})$ where $\mathrm{U}$ is a non-empty finite set of objects, called universal set and $\mathrm{R}$ be an equivalence relation defined on $\mathrm{U}$

\section{Definition 2.1.}

[11] For any approximation space, the equivalence classes induced by $\mathrm{R}$ is defined by $[x]=\{y \in U \mid(x, y) \in R\}$. For any $X \subseteq U$, the lower approximation is defined as $R_{-}(X)=\{x \in U \mid[x] \subseteq X\}$ and the upper approximation is defined as $R^{-}(X)=\{x \in U \mid[x] \cap X$ $=\varnothing\}$. The rough set corresponding to $\mathrm{X}$ is an ordered pair $R S(X)=$ $\left(R_{-}(X), R^{-}(X)\right)$.

\section{Definition 2.3.}

[6] Let $X, Y \subseteq U$. The Praba $\Delta$ is defined as $X \Delta Y=X \cup Y$, if $I W(\mathrm{X}$ $\cup \mathrm{Y})=I W(X)+I W(Y)-I W(X \cap Y)$. If $I W(X \cup Y)>I W(X)+I W$ $(Y)-I W(X \cap Y)$, then identify the new equivalence class obtained by the union of $X$ and $Y$. Then delete the elements of that class belonging to $\mathrm{Y}$. Call the new set as $\mathrm{Y}$. Now obtain $\mathrm{X} \Delta \mathrm{Y}$. Repeat the process until $I W(\mathrm{X} \cup \mathrm{Y})=I W(X)+I W(Y)-I W(X \cap Y)$.

\section{Definition 2.4.}

[6] Praba $\nabla$ of $X$ and $Y$ is denoted by $X \nabla Y$ and it is defined as $X \nabla Y$ $=\{x \mid[x] \subseteq X \cap Y\} \cup P_{X \cap Y}$, where $\mathrm{X}, \mathrm{Y} \subseteq \mathrm{U}$ and $P_{X \cap Y}$ contains those elements of $U$ whose corresponding equivalence classes are not a subset of $\cap Y$ but will have non empty intersection with $X$ and $Y$.

\section{Theorem 2.1.}

[7] For any approximation space $\mathrm{I}=(\mathrm{U}, \mathrm{R}),(T, \Delta, \nabla)$ is a semiring called the Rough semiring.

\section{Remarks 2.1.}

[7] Without loss of generality let us assume that there are nequivalence classes $\left\{X_{1}, X_{2}, \ldots, X_{n}\right\}$ in $\mathrm{U}$. Out of which there are $\mathrm{m}$ classes $\left\{X_{1}, X_{2}, \ldots, X_{m}\right\}$ (say) have cardinalities greater than one and the remaining $\mathrm{n}-\mathrm{m}$ clsses have cardinality equal to one. Note that $|\mathrm{T}|=2^{\mathrm{n}-\mathrm{m}} 3^{\mathrm{m}}$. Let $\mathrm{B}=\left\{x_{i} \mid x_{i} \in X_{i}\right.$ and $\left.\left|X_{i}\right|>1\right\}$ and $J=\{$ $R S(X) \mid X \in P(B)\}$ then $\mathrm{J}$ is an ideal of the Rough semiring called the Rough ideal . 


\subsection{Graph Theory}

\section{Definition 2.5.}

[1] Given an undirected graph $\mathrm{G}=(\mathrm{V}, \mathrm{E})$, a dominating set is a subset $\mathrm{D} \subseteq \mathrm{U}$ of its nodes such that for all nodes $\mathrm{v} \in \mathrm{V}$, either $\mathrm{v} \in$ $\mathrm{S}$ or a neighbour $\mathrm{u}$ of $\mathrm{v}$ is in $\mathrm{D}$. Number of vertices in the minimal dominating set is the domination number.

\subsection{Rough Ideal based Rough Edge Cayley Graph}

[8] In this section, we consider an approximation space $\mathrm{I}=(\mathrm{U}, \mathrm{R})$ where $\mathrm{U}$ is the non-empty finite set of objects and $\mathrm{R}$ is an equivalence relation on $\mathrm{U}$. Let $(T, \Delta, \nabla)$ be the rough semiring induced by I. Let B be the set of representative elements of $X_{i}, \mathrm{i}=$ $1,2, \ldots, \mathrm{m}$ and $\mathrm{J}$ be the rough ideal as in the previous section. We also assume that $\mathrm{M}$ is the union of none, one or more equivalence classes whose cardinality is equal to one and $M^{\prime}$ is the union of one or more equivalence classes whose cardinality is equal to one.

\section{Definition 2.6:}

[8] Rough Ideal based Rough Edge Cayley Graph denoted $G(T(J))=(V, E)$ where $V=T$ and $E=\{(R S(X), R S(Z)) \mid R S(X) \nabla R S(Y)=R S(Z), R S(Z) \in T$, $R S(Y) \in J\}$

\section{Domination Number of Rough Ideal based Rough Edge Cayley Graph}

In this section, we are going to obtain the domination number of any Rough ideal based Rough Edge Cayley Graph

\section{Theorem 3.1.}

Domination number of $G(T(J))$ is 1 .

Proof. Let $\left\{X_{1}, X_{2}, \ldots, X_{n}\right\}$ be the equivalence classes induced by $\mathrm{R}$ and without loss of generality assume that $\left\{X_{1}, X_{2}, \ldots, X_{m}\right\}, \mathrm{m} \leq \mathrm{n}$ be the equivalence classes whose cardinality is greater than one. Let $\left\{\left\{x_{1}\right\},\left\{x_{2}\right\}, \ldots,\left\{x_{m}\right\}\right\}$ be the pivot elements chosen from these equivalence classes respectively.

The Rough Ideal $J=\{R S(Y) \mid Y \in P(B)\}$ where $\quad \mathrm{B}=$ $\left\{\left\{x_{1}\right\},\left\{x_{2}\right\}, \ldots,\left\{x_{m}\right\}\right\}$.

Let $T=\{R S(X) \quad \mid X \subseteq U\}$

Consider the vertex $R S(\varnothing)$ n the Rough ideal based Rough Edge Cayley Graph $G(T(J))$.

Each element of $\mathrm{T}$ is connected to $R S(\varnothing)$ as $R S(X) \nabla R S(\varnothing)=$ $R S(\varnothing)$ where $\mathrm{RS}(\mathrm{X}) \in \mathrm{T}$.

Hence the dominating set $\mathrm{D}=\{R S(\varnothing)\}$ and hence the domination number of $G(T(J))$ is 1 .

\section{Remarks 3.1.}

To have better understanding of the Rough Ideal based Rough Edge Cayley Graph $\mathrm{G}(\mathrm{T}(\mathrm{J})$ ), instead of $\mathrm{J}$ let us consider $\widehat{J}=$ $\{R S(Y) \mid Y \in P(B), Y \neq \emptyset\}$. We obtain the Rough Ideal based Rough Edge Cayley Graph $G(T(\widehat{J}))$ by removing the edges $R S(X) \nabla R S(\emptyset)$ from $G(T(J))$.

\section{Theorem 3.2.}

Domination number of $G(T(\widehat{J}))$ is 2 .

Proof. Consider the element $R S(\varnothing)$ in the Rough Ideal based Rough Edge Cayley Graph $G(T(\widehat{J}))$.

$R S(\varnothing)$ is adjacent to all vertices of $G(T(\widehat{J}))$ except
(a) $R S\left(\left\{x_{1}, x_{2}, \ldots, x_{m}\right\}\right)$ where $x_{i} \in B$
(b) $R S\left(\left\{x_{1}, x_{2}, \ldots, x_{m}\right\} \cup M^{\prime}\right)$ where $x_{i} \in B$
(c) $R S\left(\left\{x_{1}, x_{2}, \ldots, x_{m}\right\} \cup M\right)$ where $x_{i} \in B$.
(d) $R S\left(Q_{m} \cup M\right)$

But the element $R S\left(\left\{x_{1}, x_{2}, \ldots, x_{m}\right\}\right)$ is adjacent to $R S\left(\left\{x_{1}, x_{2}, \ldots, x_{m}\right\} \cup M^{\prime}\right), R S\left(\left\{x_{1}, x_{2}, \ldots, x_{m}\right\} \cup M\right)$ and $\quad\left(Q_{m} \cup\right.$ $M)$. Hence $\mathrm{D}=\left\{R S(\emptyset), R S\left(\left\{x_{1}, x_{2}, \ldots, x_{m}\right\}\right)\right\}$. Hence the Domination number of an Rough Ideal based Rough Edge Cayley graph $\mathrm{G}(\mathrm{T}(\widehat{J}))$ is 2 .

\section{Example 4.1.}

Let us consider the approximation space $(\mathrm{U}, \mathrm{R})$ where $U=$ $\left\{x_{1}, x_{2}, x_{3}, x_{4}\right\}$.

The equivalence classes induced by $\mathrm{R}$ are: $X_{1}=\left\{x_{1}, x_{3}\right\}, X_{2}=$ $\left\{x_{2}, x_{4}\right\}$.

$T=\left\{R S(\emptyset), R S(U), R S\left(X_{1}\right), R S\left(X_{2}\right), R S\left(\left\{x_{1}\right\}\right), R S\left(\left\{x_{2}\right\}\right)\right.$,

$\left.R S\left(X_{1} \cup\left\{x_{2}\right\}\right), R S\left(\left\{x_{1}\right\} \cup X_{2}\right), R S\left(\left\{x_{1}\right\} \cup\left\{x_{2}\right\}\right)\right\}$.

If $B=\left\{x_{1}, x_{2}\right\}$

then $J=\left\{R S(\varnothing), R S\left(\left\{x_{1}\right\}\right), R S\left(\left\{x_{2}\right\}\right), R S\left(\left\{x_{1}, x_{2}\right\}\right)\right\}$

The vertices of $G(T(J))$ are the elements of $T$.

Each element $R S(X)$ in $T$ is connected to $R S(X) \nabla R S(Y)$, where $R S(Y) \in J$.

For example, $R S\left(X_{1}\right) \nabla R S\left(\left\{x_{1}\right\}\right)=R S\left(\left\{x_{1}\right\}\right)$, where $R S\left(X_{1}\right) \in$ $T$ and $R S\left(\left\{x_{1}\right\}\right) \in J$. Hence there is an edge between $R S\left(X_{1}\right)$ and $R S\left(\left\{x_{1}\right\}\right)$.

$R S\left(X_{1}\right) \nabla R S\left(\left\{x_{2}\right\}\right)=R S(\varnothing)$. Hence there is an edge between $R S\left(X_{1}\right)$ and $R S(\varnothing)$.

Using the similar argument by considering all the elements of $\mathrm{T}$ as vertices, the Rough Ideal based Rough Edge Cayley Graph $G(T(J))$ can be obtained and is shown in Figure:1.

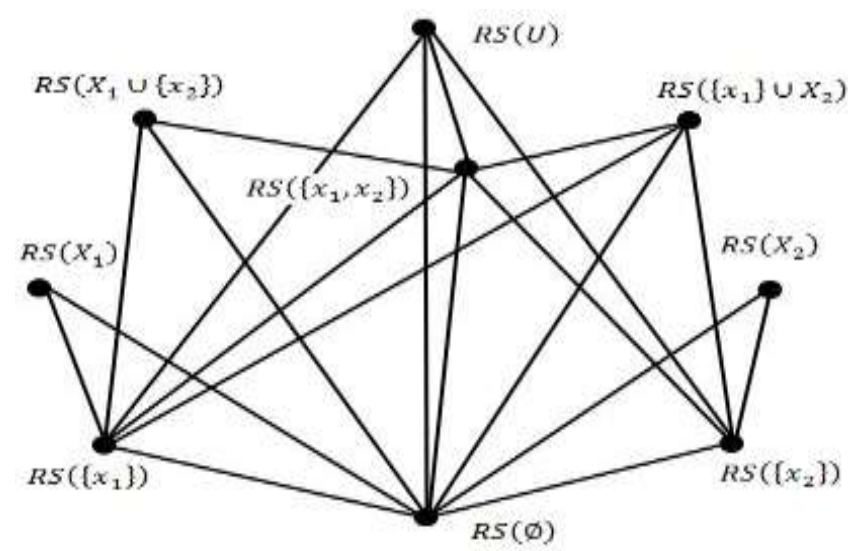

Figure1: $\mathbf{G}(\mathrm{T}(\mathrm{J}))$ when $\mathrm{n}=2$ and $\mathrm{m}=2$

From figure: 1 , we observe that the dominating set of $G(T(J))$ is D $=\{R S(\emptyset)\}$. Hence the domination number of this Rough Ideal based Rough Edge Cayley Graph $G(T(J))$ is 1 .

The Rough Ideal based Rough Edge Cayley graph $G(T(\widehat{J}))$ is shown in figure: 2 and we observe from the figure that the dominating set of $G(T(\widehat{J}))$ is $\mathrm{D}=\left\{R S(\varnothing), R S\left(\left\{x_{1}, x_{2}\right\}\right)\right\}$. Hence the domination number of this Rough Ideal based Rough Edge Cayley Graph $G(T(\widehat{J}))$ is 2 .

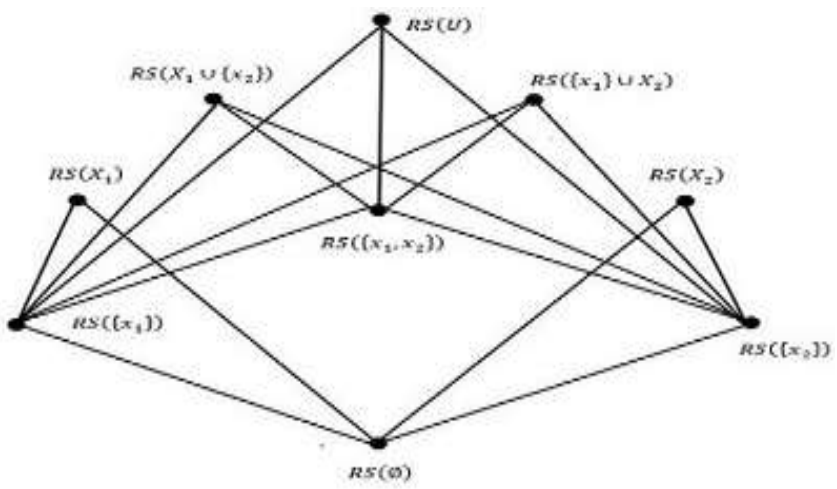

Figure2: $\mathrm{G}(\mathrm{T}(\widehat{J}))$ when $\mathrm{n}=2$ and $\mathrm{m}=2$ 


\section{Domination Number of a Communication Network}

A communication network can be viewed as a Rough Ideal based Rough Edge Cayley graph. Let $\mathrm{V}$ be the underlying set of nodes and these nodes are classified into disjoint sets according to their priority. The elements of $\mathrm{T}$ are the subsets corresponding to each subset of $\mathrm{V}$ containing the priority classes that are completely contained in that set and the priority classes having non empty intersection. The pivot set contains the representatives from each of the priority classes subject to the condition that it should have more than one element. The Rough Ideal is closed subset of $\mathrm{T}$ containing all the representatives of the priority classes. Now our dominating set is the minimum number of elements of $\mathbf{J}$ such that these nodes are reachable from every other nodes. The element of $\mathrm{J}$ that contains union of all the pivot elements are reachable from every other nodes at a distance of 1 or 2 except the classes with no pivot elements. The classes with no pivot elements are connected only to the pendant vertex. These can be the minimum number of elements that form the dominating set.

\section{Conclusion}

From the literature survey we observe that the Cayley graph has been defined on a Group, but in this paper we made an attempt of defining a Cayley graph corresponding to the Rough Ideal $\mathrm{J}$ of the Rough semiring $(T, \Delta, \nabla)$. The Domination number of the Rough Ideal based Rough Edge Cayley graphs $G(T(J))$ and $G(T(\widehat{J}))$, where $\widehat{J}$ contains the non-trivial elements of $\mathrm{J}$ are obtained and illustrated through examples. A communication network has been interpreted as the Rough Ideal based Rough Edge Cayley graph and the dominating set for it is obtained.

\section{Acknowledgement}

The authors thank the management and the Principal, SSN Institutions for the completion of this paper and providing encouragement and support to carry out this research.

\section{References}

[1] J.A.Bondy and U.S.R.Murty, (1976), Graph theory with applications, University of Waterloo, Ontario, Canada.

[2] B. Davvaz, Roughness based on fuzzy ideals, (2006), Information Sciences 176, 2417-2437.

[3] Jinkun Chen, Jinjin Li, (2012), An application of rough sets to graph theory, Information Sciences, 201, 114-127.

[4] A.Manimaran, B.Praba and V.M.Chandrasekaran, Characterization of Rough Semiring, Afrika Mathematika, DOI 10.1007/s13370017-0495-7 (2017).

[5] Meilian Liang, Binmei Liang, Linna Wei and Xiadong Xu, (2011) Edge rough graph and its applications, $8^{\text {th }}$ International conference on Fuzzy systems and Knowledge discovery, $\mathrm{Pg}: 335-338$.

[6] B. Praba and R. Mohan, (2013), Rough Lattice, International Journal of Fuzzy Mathematics and Systems, Volume 3, Number 2, pp. $135-151$

[7] B.Praba, V.M.Chandrasekaran and A.Manimaran, (2015), Semiring on Rough sets, Indian Journal of Science and Technology, Vol. 8(3), 280-286.

[8] B.Praba and Benazir Obilia.X.A, (2017), Domination number of Rough Ideal based rough Edge Cayley Graph through Category graph, International Journal of Pure and Applied Mathematics, Volume 117, No. 9, 187-191.

[9] V.Selvan and G.Senthil Kumar, (2012), Rough ideals in Semirings, International Journal of Mathematics Sciences and Applications, Vol. 2, No. 2.
[10] M.H. Shahzamanian, M. Shirmohammadi, B. Davvaz, (2010), Roughness in Cayley Graphs, Information Sciences 180, 33623372.

[11] Zdzislaw Pawlak, (1982), Rough Sets, International Journal of Computer and Information Sciences, Vol.11, 341-356. 